\title{
Becoming Visionary: Evaluation of a Comprehensive Eye Exam Clinic Within a Geriatric Rehabilitation Setting
}

\author{
Jennifer Klein PhD OT (C) Comb Honors BA (Gerontology and Sociology) \\ Cathryn Schmidt RN, MN \\ Aleem Bandali OD \\ Ross McKenzie OD \\ Correspondence may be directed to: jennifer.klein@ualberta.ca
}

\begin{abstract}
In the older adult population, visual impairments are highly prevalent, but largely undiagnosed. Research has shown that such visual impairments increase the risk of falls, depression, and mortality amongst older adults. Studies are lacking that examine the impact of implementing an eye exam clinic within a hospital setting. The goal of this proposed study was to evaluate the feasibility of having community optometrists offer a Comprehensive Eye Exam (CEE) Clinic for detecting vision loss within a geriatric rehabilitation setting. Findings revealed $83 \%$ of participants were diagnosed with detectable vision problem of which more than half were previously undiagnosed. One third of participants had not seen an eye specialist within the past two years. The CEE Clinic was implemented with minimal expenses, low workload burden on staff, and detected significant vision issues among many participants.
\end{abstract}

\section{Introduction}

Changes in vision are common within the older adult population. Findings indicate that visual impairment amongst older adults doubles the incidence of falls and mortality rates, triples the incidence of depression, and quadruples the incidence of hip fractures. ${ }^{1-3}$ In contrast, research has found the improvement in visual function resulting from treatment of vision disorders is accompanied by improvement in life satisfaction, mobility, mental health, home activities, and community activities. ${ }^{4}$ Estimates indicate $20-50 \%$ of seniors have undetected reduced vision. ${ }^{5}$ Of those who have visual impairment, over $50 \%$ of impairments are due to easily correctable conditions. ${ }^{6}$ Most of these ocular conditions such as refractive errors and cataracts are treatable, especially if caught early, thus emphasizing the importance of screening programs. $^{7}$

Studies are lacking that examine the feasibility and impact of implementing a screening program within a hospital setting, making it difficult to draw firm conclusions about whether hospitals should implement screening programs for their older patients in hopes of minimizing visual deficits amongst their older patients.

\section{Study Goals and Objectives}

Goal: The goal was to evaluate the feasibility of having community optometrists offer a Comprehensive Eye Exam (CEE) Clinic for detecting vision loss within a geriatric rehabilitation setting.

Objectives: The first objective was to determine outcomes related to implementing the program: number of patients assessed, prevalence of patients with visual concerns and deficits, frequency of various visual diagnosis, number or patients referred for further visual assessment; and number of patients who booked follow-up appointments.

The second objective was to examine personal experiences with the pilot: enablers (i.e., things which assist the pilot in being successful) and challenges as viewed by the staff, patients, and community optometrists involved in the CEE Clinic.

\section{Methods}

Design: This was a descriptive feasibility mixed-methods study. Feasibility studies are used to determine whether an intervention is appropriate for further testing. The study site consisted of two geriatric rehabilitation units, each with 36 
beds at a rehabilitation hospital in Western Canada. Generally, patients on these units are admitted from acute care hospitals. The sample comprised 55 participants. Patients who reported having had an eye exam within the last year were excluded from the study to reduce redundant health care provision. The CEE clinics took place April-July, 2014. The Health and Research Ethics board of the local university approved this study.

Recruitment: All patients admitted to the units during the clinic time period were asked if they would like to receive further information about the study. If patients agreed, a research assistant met with them to discuss the study and determine eligibility. One hundred and thirty-eight patients were approached, of which 33 patients stated they had already seen an eye specialist within the past year, 5 had significant cognitive impairments limiting their ability to follow directions, 55 accepted to participate, and 45 declined. Reasons for declining included: discharged prior to clinic appointment $(n=18)$, admitted to isolation unit $(n=7)$, or not interested $(n=20)$.

\section{Intervention}

Objective 1. A community optometrist performed a comprehensive eye exam with a portable community evaluation kit in an assessment room within the hospital. The comprehensive eye exam comprised of a case history, acuity test using the Snellen Eye Test, neurological assessment with standard non-computerized tests (pupil reaction, confrontation visual fields, eye muscle movements, etc.), prescription assessment, and eye pressure test. The eye exam was performed in a well-lit room. For the visual acuity exam, the Snellen eye chart was placed $10 \mathrm{~m}$ from the patient. The left eye was covered and the right eye was evaluated in a progressive fashion and then the left eye was independently evaluated in the same progressive manner. The acuity test focused on the habitual visual acuity (i.e., the vision participants present with at time of examination with or without glasses) as the pilot was trying to analyze current functional level of vision. Intraocular pressure (IOP) was tested using the Tonopen, a small handheld, compact, portable applanation tonometer. The Tonopen has been shown to be a valid and reliable tool to test IOP. ${ }^{7}$ Intraocular pressure testing can help identify presence of glaucoma.

Approximately 8 patients were seen per 3 -hour clinic, which was held one day every 2 weeks with one of two community optometrists. Patients with detected eye disease were referred for further eye services. If the optometrist recommended a visit to an ophthalmologist, then the patient's doctor was informed of these findings and the optometrist made a phone call to set up a referral assessment with an ophthalmologist at the nearby acute care hospital.

Three to 4 months after the CEE clinic, a follow-up phone call was made by the research assistant to all participants who were recommended to seek further visual evaluation, to determine whether they pursued a follow-up appointment.

Objective 2. Personal experiences regarding the pilot clinic were gathered in both written and oral format. Written feedback was obtained from the two optometrists to examine their experiences in taking part in the CEE Clinic. Probing questions were asked such as: "Can you tell me about your experiences with respect to the implementation the Comprehensive Eye Exam Clinic?" During the clinic and follow-up phone calls, staff and patients' comments regarding their experiences with the clinic were also recorded.

\section{Analysis}

Research Objective 1: Patient data was entered into the computer program using Statistical Package for the Social Sciences (SPSS). Descriptive statistics such a means, standard deviations, and percentages were analyzed relating to patient demographics and data relating to visual exams.

Research Objective 2: Content analysis was used to analyze the data. Themes were refined as patterns emerged.

\section{RESULTS}

\section{Patient characteristics}

The patient population targeted comprised of older adults admitted for geriatric rehabilitation. Table 1 includes demographic characteristics of the 55 participants.

Table 1. Characteristics of the Study Sample

\begin{tabular}{|l|l|}
\hline Age (mean, SD) & $80.5(8.44)$ \\
\hline Sex (female, $n \%$ ) & $31(56.4)$ \\
\hline Length of Stay in days (mean, SD) & $42(20.3)$ \\
\hline
\end{tabular}

\section{Screening characteristics}

The mean length of time since the last eye exam was 3.4 years $(\mathrm{SD}=3.8$ years). The range was 6 months to 20 years. Even though patients were only included in the pilot if they told the recruiter they had not seen an eye specialist within the year, several later told the OD that they indeed had seen an eye specialist within the year. Thirty-five people (63.6\%) reported having seen an eye specialist within the past two years. Table 2 illustrates in greater detail the range of time since last eye exam. 
Table 2. Length of Time Since Last Eye Exam

\begin{tabular}{|l|l|}
\hline$\leq 12$ months $(n, \%)$ & $18(32.7)$ \\
\hline $13-24$ months $(n, \%)$ & $35(63.6)$ \\
\hline 25 months to 5 years $(n, \%)$ & $7(12.7)$ \\
\hline$>5$ years but $<10$ years $(n, \%)$ & $13(23.6)$ \\
\hline 10 years or more $(n, \%)$ & $6(10.9)$ \\
\hline
\end{tabular}

Table 3. Detected Eye Conditions

\begin{tabular}{|c|c|}
\hline $\begin{array}{l}\text { Cataracts }(n, \%) \\
\quad \text { Previously undetected }\end{array}$ & $\begin{array}{c}20(40) \\
12\end{array}$ \\
\hline $\begin{array}{l}\text { Macular degeneration }(n, \%) \\
\text { Previously undetected }\end{array}$ & $\begin{array}{ll}9 & (16) \\
& 4\end{array}$ \\
\hline $\begin{array}{l}\text { Glaucoma }(n, \%) \\
\text { Previously undetected }\end{array}$ & $\begin{array}{ll}5 & (9) \\
& 3\end{array}$ \\
\hline $\begin{array}{l}\text { Blepharitis/ Dry eye syndrome }(n, \%) \\
\text { Previously undetected }\end{array}$ & $\begin{array}{ll}8 & (15) \\
& \text { unknown }\end{array}$ \\
\hline $\begin{array}{l}\text { Other visual problems (e.g., acuity changes) }(n, \%) \\
\text { Previously undetected }\end{array}$ & $\begin{array}{l}7 \text { (13) } \\
\text { unknown }\end{array}$ \\
\hline $\begin{array}{l}\text { Retinal hemorrhage }(n, \%) \\
\text { Previously undetected }\end{array}$ & $\begin{array}{c}1(2) \\
0\end{array}$ \\
\hline
\end{tabular}

Figure 1. Visual acuity results with Snellen Chart.

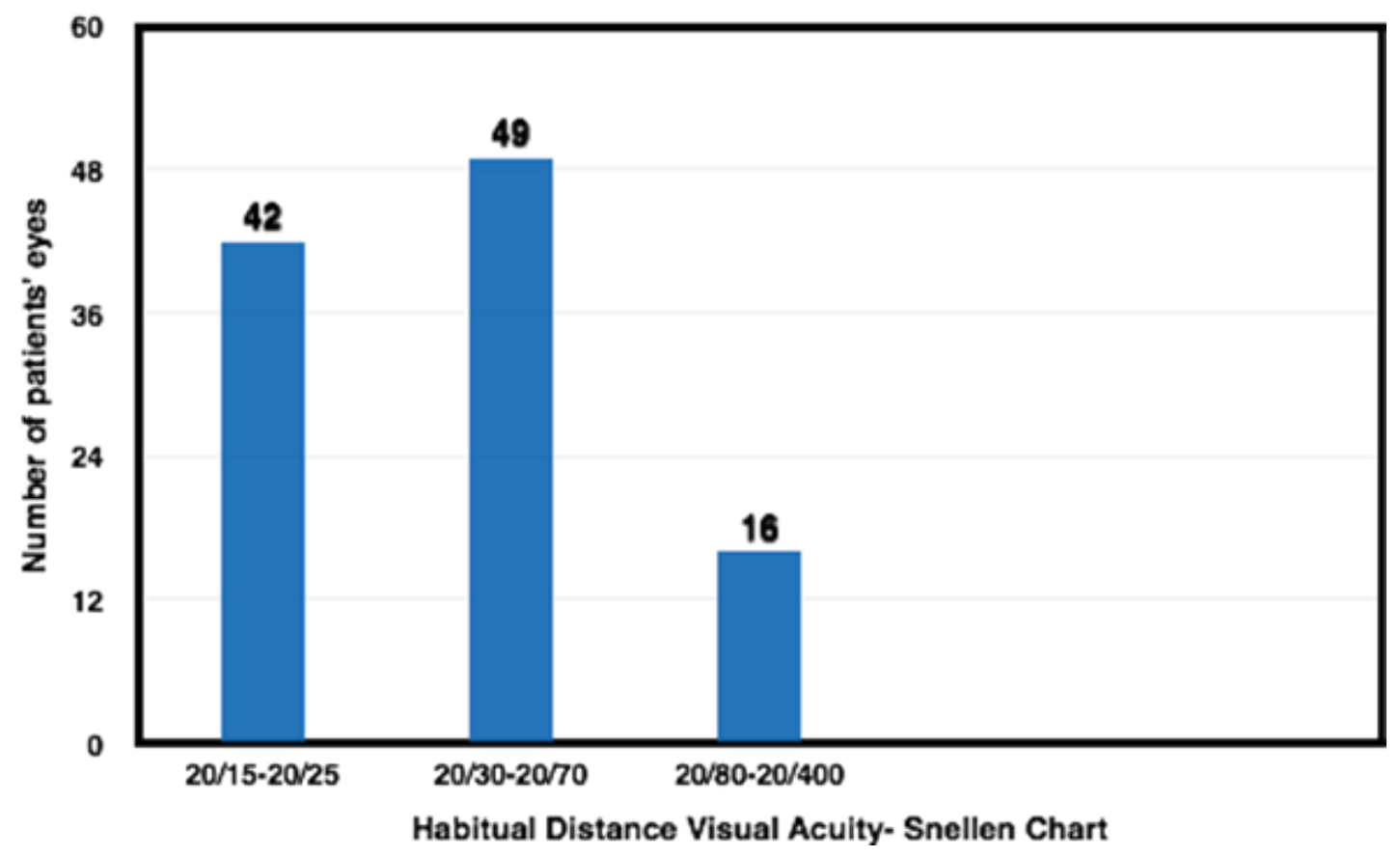

Vision screening and eligibility for referral

At the beginning of the eye exam, patients were asked whether they had any visual concerns. Twenty-one participants $(38 \%)$ replied that they did indeed have a concern (e.g., blurred vision, "not seeing too well," was already told that cataracts were starting to develop). Upon completion of the examination, 46 participants $(83 \%)$ were deemed to have detectable vision problems. The most common finding was the presence of cataracts (20 participants). Of the 35 eye diseases detected by the community optometrists, 19 (54.3\%) were previously undiagnosed. Table 3 outlines the various detected eye conditions.

Testing patients' left and right eyes for their habitual visual acuity, $59 \%$ of participants presented with mild to severe visual impairment of 20/30 or greater. Fifteen percent of participants presented with moderate or severe visual impairment of 20/80 or greater. Figure 1 shows the results of the clinical examination using the Snellen Chart.

Using the Tonopen, the mean IOP was $15 \pm 4.01 \mathrm{~mm} \mathrm{Hg}$. Only three participants presented with an IOP of greater than $21 \mathrm{~mm} \mathrm{Hg}$. 


\section{Referrals}

Twenty-seven participants (49\%) were recommended to pursue further follow-up. We examined the willingness of these participants to book follow-up eye care services after the CEE in the hospital. When the optometrists discussed with certain participants the need for further follow-up after their examination, all participants showed interest in pursuing follow-up. Three to four months after discharge, the research assistant called each of these 27 participants who were recommended for further examination. Twelve participants (44\%) had already followed up independently to book their appointment prior to the research assistant's initial phone call. Fourteen of 27 older adults (52\%) had not yet made an appointment when the research assistant contacted them. One patient could not be reached by phone. For many participants, the three-month follow-up phone call served as a reminder for them to book their appointments.

Of the twenty-seven participants who were advised to have further follow-up, 16 were recommended to see an optometrist and 11 were referred to an ophthalmologist. Table 4 denotes the patient follow-through with these recommendations. Three to four months after discharge, there was moderate willingness amongst participants (66\%) to pursue further follow-up.

Patients who were referred for, but did not attend a second exam, were contacted and asked why they did not follow-up. Barriers included: participants forgetting to book appointment, difficulty finding transportation and/or a family member to get them to an appointment, and too many other health problems and/or doctor appointments that needed to be dealt with prior to making an appointment for a follow-up eye exam. Several said they "could not be bothered" to book the appointment.

\section{Experiences}

\section{Staff responses to the program}

The CEE pilot clinic was well received by staff. Nursing staff, social workers and unit clerks reported that the clinic did not add to their workload. An onsite CEE clinic was viewed as a convenient way to have patients' eyes examined, as patients

Table 4. Patient Follow-Through with Follow-Up Appointments

Referral to see an optometrist $n=16$

\begin{tabular}{|l|l|}
\hline Booked appointment & 9 \\
\hline Not interested in booking & 4 \\
\hline "I will call, but haven't yet" & 3 \\
\hline
\end{tabular}

Referral to see an ophthalmologist $n=11$

\begin{tabular}{|l|l|}
\hline Booked appointment & 9 \\
\hline Not interested/did not answer phone & 2 \\
\hline
\end{tabular}

did not require being transported to another hospital site. One therapist shared that the CEE clinic was "another tool to the puzzle" to assess patients' functional mobility related to visual acuity. Another commented that having access to an optometry assessment "compliments the comprehensive assessment and perhaps can look at the cause of the patient's dizziness and instability."

\section{Patients' experiences}

Patients reported being pleased with the services and convenience of the clinic being on-site. Several challenges were mentioned regarding patients' lack of regular eye care in the community. The biggest challenges were finding transportation to get to the eye exam and knowing whom to contact to get their eyes examined.

Several patients spoke of being overwhelmed with their other health conditions and numerous medical appointments, thus making it harder to pursue eye appointments saying they were too busy with so many appointments.

One gentleman with glaucoma shared:

"As soon as I can fix this (walking) then I can focus on my eyes. But right now I need to focus on my legs. This study reminded me to get back on track to having my eyes checked every six months. I let it (vision checkups) go because I had health problems."

Some participants and their family members were frustrated by the fact that they needed to do a second more indepth evaluation at an optometry clinic once discharged. They were hoping the in-house clinic would be able to diagnose visual problems immediately and provide appropriate eyewear prior to leaving the clinic. However, as a temporary clinic, the optometrists did not have the equipment to accurately measure refraction.

\section{Optometrists' experiences}

The clinic was well received by the two community optometrists. One reported:

"I think the idea of having a primary eye care clinic within the hospital is an excellent idea. I would love to see optometry and vision care incorporated into the treatment received by patients. I was honestly amazed at the wide range of visual abilities.... Many of them had fairly extensive vision problems, and this was only in one 
small sub-section of the hospital. Some of these problems were longstanding, but others were more active and required treatment. Some were less urgent like cataracts, but still these can impact personal mobility. Many other vision care problems including visual field loss might impact how a patients' rehab is performed."

The optometrists noted several challenges. Their services were limited due to the temporary nature of the screening clinic. Optometrists were not able to accurately measure refraction with their travel eye exam kit. Nor could they complete visual field tests for patients who would benefit (e.g., patients who had experienced a stroke). Another challenge was the larger than anticipated amount of paperwork required in the hospital compared to working in the community. The size of one of the two assessment rooms also posed a problem. It was 10 feet long by 9 feet wide, making it difficult to navigate patients in wheelchairs.

\section{Discussion}

Findings from this pilot study suggest it is feasible to implement a Comprehensive Eye Exam clinic for older adults within a hospital setting. The CEE clinic was implemented with minimal expenses, low workload burden on staff, and detected significant vision issues among many participants. The hospital and patients of all ages could benefit from having a CEE clinic.

It is concerning that more than one third of participants had not had their eyes examined in the past 2 years. The Canadian Association of Optometrists (CAO) recommends adults aged 65 years or older undergo an eye examination annually. ${ }^{8}$ The Canadian Ophthalmological Society recommends comprehensive eye exams every two years for people aged 65 and older who have no risk factors. ${ }^{9}$ More concerning is the fact that $10 \%$ of participants had not had an eye examination in over 10 years. There were inconsistencies between participants reporting having visual concerns at the outset of the eye exam (38\%) and the number of participants who were diagnosed with a detectable vision problem (83\%). This is supported in the literature. The U.S. Preventive Services Task Force ${ }^{10}$ reported patient self-reports are not as accurate as undergoing comprehensive eye exams in determining visual problems. Individuals under report visual concerns, in part because they progress slowly.

The biggest challenge to implementing a temporary screening was the requirement of many patients to follow through with an external second appointment at a later date. In this study, the attrition rate was quite high for these follow-up appointments. Only $44 \%$ of participants followed up with a second booking, and many of these were made by the optometrist who booked the appointments for the ophthalmologist. Even with a staff member performing reminder phone calls, the booking rate increased modestly to $66 \%$ of participants. This appears to be resource intensive.

In order to alleviate this barrier to providing optimal eye health for seniors, a permanent in-house eye care clinic could allow for the accommodation of specialized equipment. This would enable the testing for refraction and eyeglass prescription. Fixed costs of such a clinic would largely be related to start-up costs and costs for equipment and overhead. Fixed costs would include: a computer to increase efficiency and charting, equipment stands and slit lamps that would be able to accommodate both individuals with and without mobility issues, an eye chart, occluder, hand held equipment, auto refractor, diagnostic drops, tissue, exam charts, prescription pads, sink to wash up, and antiseptic cleaner. Start up of such a clinic in-house is estimated at $\$ 50-75,000$.

In addition, the clinic could benefit from a full time clinic administrator and part-time or full time optometry assistant to ensure continuity of care, if multiple doctors were used and to assist on clinic days. Optometrists would bill the province for their eye exam services, thus causing no additional salaried costs to the hospital.

Such a clinic could enable further in-depth evaluations of patients, which can assist the patient and their health care team in providing additional information regarding patients' visual abilities impacting the teams' comprehensive assessments and interventions. Such a clinic could also minimize redundancy of follow-up evaluations outside the clinic for those with concerns regarding acuity and prescription changes; as well as enabling participation of those seniors who have challenges finding transportation to access community clinics.

This pilot study was a pioneer of its kind, as it was the first study involving optometrists performing hospital-based comprehensive eye exams within the province of Alberta. Typically ophthalmologists have done these hospital-based exams. However, the cost burden on the health system is significantly higher when ophthalmologists, opposed to optometrists, perform these initial comprehensive eye exams. ${ }^{11}$ It is timely to explore the possibility of optometrists performing initial in-house comprehensive eye exams. Doing so enables the maximization of the strength of both professions, thus providing more efficient utilization of health care resources, 
as well as enabling ophthalmologists to be available to perform medical services more appropriate to their scope of practice (e.g., eye surgeries), all the while providing timely and much needed access to eye care for older patients.

This program fits well with the hospital's current mission which is to "provide excellence and innovation in the delivery of patient and family-centered care and leading the provision of specialized rehabilitation services," as well as "collaborating with a wide range of partners to address the needs of patients and the community and building a strong, integrated system of health care delivery." Considering the scientific evidence showing links between treating visual disorders and improved life satisfaction, mobility, mental health, and quality of life, ${ }^{4,11}$ an optometrist-lead inpatient eye clinic would truly be a way to partner with the local community while delivering excellence in patient care.

The potential for future community optometrists to participate in such a clinic appears promising. Kergoat et al recently reported in a national study of Canadian optometrists that $41 \%$ of Canadian optometrists would consider seeing older frail patients outside their office. ${ }^{12}$ From this group, $41 \%$ would accept doing so half day per month and $8.6 \%$ would be willing to offer up to half a day per week.

\section{Clinical Relevance}

This study addresses an important gap in the literature and practice environment in eye exams related to the inpatient geriatric population. Vision impairment is consistently associated with decreased functional capacity and quality of life in older persons, including the ability to live independently, with more severe vision impairment associated with greater negative effects. ${ }^{10}$ While eye diseases pose significant risk to seniors, optometrists have proven their ability to examine and improve the eye health of seniors. Findings can help strengthen partnerships between the local health care community and hospitals.

Data and outcomes related to implementing an optometristlead CEE Clinic for geriatric inpatients are available for the first time. In an era where there are significant fiscal restraints when providing health care services, examining new, creative and fiscally conservative health services to examine the feasibility of such a clinic is opportune. Linking community health professionals with the local hospital is one innovative way to potentially minimize cost while providing optimal health care for our seniors. Results are relevant to all tertiary rehabilitation hospitals in Canada, and can be of considerable interest to other national and international facilities that operate programs for older adults.

\section{References}

1. National Coalition for Vision Health. Vision loss in Canada. National Coalition for Vision Health; 2011. Available at: http://www.visionhealth.ca/ news/Vision\%20Loss\%20in\%20Canada\%20-\%20Final.pdf.

2. Salonen L, Kivela, SL. Eye diseases and impaired vision as possible risk factors for recurrent falls in the aged: A systematic review. Curr Gerontol Geriatr Res 2012;(2);71-81.

3. Brown R, Lewis B, and Anne E. Visual impairment and quality of life among older adults: An examination of explanations for the relationship. J Gerontol, Series B: 2011;66B(3):364-73.

4. Smeeth L, Fletcher AE, Hanciles S, et al. Screening older people for impaired vision in primary care: cluster randomised trial. Br Med J 2003;327:1027-31.

5. Evans J, Smeeth L, and Fletcher A. Vision screening. Br J Opthomol 2009;93(6):704-705.

6. Carcenac G, Hérard ME, Kergoat MJ, et al. Assessment of visual function in institutionalized elderly patients. J Am Med Dir Assoc 2009;10:45-9.

7. Bhartiya S, Bali SJ, James M, et al. Test retest variability of TonoPen AVIA. Ind J Ophthalmol 2013;61(3):129-31. doi:10.4103/0301-4738.109384.

8. Canadian Association of Optometrists. Public policy: Frequency of eye examinations. CAO; 2013. Available at: https://opto.ca/document/frequencyof-eye-examinations.

9. Canadian Ophthalmological Society. When should you see an Ophthalmologist? 2014. Available at: http://www.cos-sco.ca/vision-healthinformation/when-should-you-see-an-ophthalmologist.

10. U.S. Preventive Services Task Force. Screening for impaired visual acuity in older adults: Recommendation statement. Am Fam Phys 2011;15 (83):185-86.

11. Soroka M, Reis L, Krumholz D, et al. Alternative arrangements for the delivery of eye care services within staff model managed care organizations. Optometry 2003;74(11):711-26.

12. Kergoat H, Leach SJ, Faucher C, et al. Primary eye care services offered to older adults. Eur Geriatr Med 2015;6(3):241-44. 